\title{
Rail-to-Rail Timer-based Demodulator for AM Sensor Signals
}

\author{
Ferran Reverter
}

\begin{abstract}
This paper proposes a novel timer-based demodulator for low-frequency amplitude-modulated (AM) sensor signals with a rail-to-rail operating range. The demodulator extracts the amplitude of the AM signal by measuring the period of a reference signal that is altered by the AM signal itself, as already suggested in a previous paper. The rail-to-rail operation, which is the main contribution of the novel circuit, is achieved by simply but cleverly incorporating a multiplexer that enables the comparison between the two signals (reference and $A M)$ just at the beginning and at the end of the period measurement. This new topology offers an operating range that is up to more than four times wider than that reported in the literature. The input-output characteristic in such a wider operating range is not linear, but it can be accurately modelled by a second-degree polynomial.
\end{abstract}

Index Terms - AM signal, demodulator, digital timer, rail-torail circuit, sensor interface electronics.

\section{INTRODUCTION}

$\mathrm{R}$ EADING sensors through a time-to-digital converter (TDC) instead of an analog-to-digital converter (ADC) has several advantages, for instance: (1) in microcontroller-based designs, the current consumption of an embedded TDC is clearly lower than that of an ADC; and (2) in integrated designs, the layout area occupied by a TDC is one or two orders of magnitude smaller than that required by an ADC. Accordingly, timer-based circuits for resistive [1]-[3], capacitive [4], [5], inductive [6], [7], and voltage-output [8], [9] sensors have been extensively analyzed and developed in the last decade. For the case of voltage-output sensors, usually it is assumed that the sensor provides a quasi-static analog output voltage [8], [9]. The only attempt to measure AM sensor signals with a carrier frequency in the range of kilohertz through a digital timer was suggested in [10]. However, the proposed circuit had limitations in terms of operating range. Under certain operating conditions, the amplitude of the AM signal had to be significantly lower than the supply voltage $\left(V_{\mathrm{DD}}\right)$ of the circuit.

Based on the concept introduced in [10], this paper proposes a novel timer-based demodulator for AM sensor signals with a wider operating range. The proposed circuit is

Manuscript received Month xx, 2xxx; revised Month xx, xxxx; accepted Month x, xxxx. This work was supported by the Spanish Ministry of Economy and Competitiveness and the European Regional Development Fund under project TEC2016-76991-P.

Ferran Reverter is with the Department of Electronic Engineering, Universitat Politècnica de Catalunya - BarcelonaTech, Castelldefels (Barcelona), 08860, Spain (e-mail: ferran.reverter@upc.edu). able to demodulate signals with an amplitude that can swing all the way between the supply voltage levels and, for this reason, it is called rail-to-rail timer-based demodulator. As in [10], the demodulation is carried out without using a rectifier, a mixer, a low-pass filter, or an analog-to-digital converter, thus resulting in a low-cost design solution.

\section{PREVIOUS TOPOLOGY}

The timer-based demodulator presented in [10] extracts the amplitude of the AM sinusoidal signal by measuring the period of a reference triangular signal that suffers from interference effects [11] caused by the AM signal itself. This period measurement undergoes a deviation that carries information about the amplitude of the AM signal whenever an appropriate relation between the carrier frequency $\left(f_{\mathrm{c}}\right)$ of the AM signal and the frequency of the triangular signal $\left(f_{0}\right)$ is chosen. The amplitude of the deviation is zero when $f_{\mathrm{c}} / f_{0}=m$, whereas it is maximum when $f_{\mathrm{c}} / f_{0}=m+0.5, m$ being any positive integer including the zero. In order to have the highest effects of the AM signal on the period measurement, the case $f_{\mathrm{c}} / f_{0}=m+0.5$ should be selected. However, if $f_{\mathrm{c}} / f_{0}$ is exactly equal to $m+0.5$, the set of period measurements shows an alias component at $0 \mathrm{~Hz}$, which means that the same value of period is repeatedly measured. This can be avoided using $f_{\mathrm{c}} / f_{0}=(m+0.5)(1+\Delta)$ with $|\Delta|<<1$, thus generating a low-frequency alias component at $f_{\mathrm{c}} \cdot|\Delta|$. In such conditions, the amplitude $\left(A_{\mathrm{s}}\right)$ of the AM signal can be estimated by computing the standard deviation (STD) of the set of period measurements carried out, at least, during one period of the alias component.

The models in [10] were developed by analyzing the voltage crossings between the triangular signal and the AM signal, the latter being superimposed on the dc level of $V_{\mathrm{DD}} / 2$. Two models were proposed: a small-signal (SS) model that is valid for low values of $A_{\mathrm{s}}$ and predicts a linear relation between $A_{\mathrm{s}}$ and STD, and a large-signal (LS) model that is useful for higher values of $A_{\mathrm{s}}$ and suggests a non-linear relationship. These models assume that in a single period of the triangular signal we only have: 1) a first positive crossing that starts the period measurement, 2) a negative crossing, and 3) a second positive crossing that ends the measurement, where a positive (negative) crossing is when the triangular signal becomes higher (lower) than the AM signal. The previous assumption, however, cannot be ensured for high values of both $A_{\mathrm{s}}$ and $f_{\mathrm{c}} / f_{0}$, thus causing aberrant period measurements that cannot be employed in the calculation of STD. For example, at $V_{\mathrm{DD}}=5 \mathrm{~V}$, the maximum $A_{\mathrm{s}}$ that can be demodulated without having aberrant measurements is $6.37 \mathrm{~V}$, $2.12 \mathrm{~V}$, and $1.27 \mathrm{~V}$ for $f_{\mathrm{c}} / f_{0} \approx 0.5,1.5$, and 2.5 , respectively. 


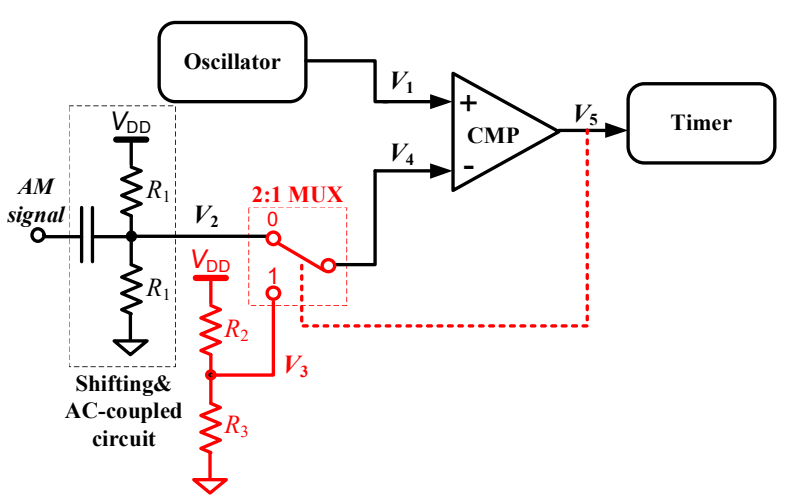

Fig. 1. Proposed rail-to-rail timer-based demodulator for AM sensor signals. The novel circuitry, in comparison with [10], is highlighted in red.

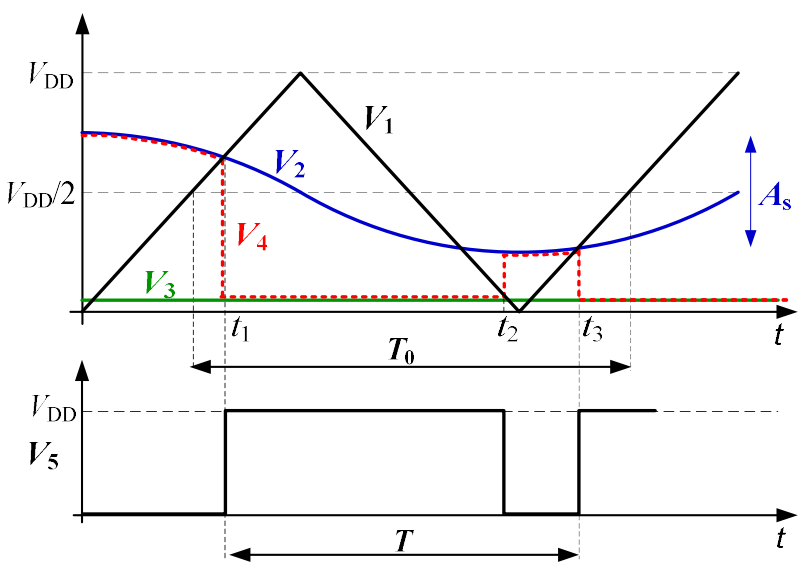

Fig. 2. Waveform of the main signals from the circuit in Fig. 1 .

\section{NOVEL TOPOLOGY}

Fig. 1 shows the novel topology of the timer-based demodulator, which is composed of: 1) an oscillator providing a triangular-wave voltage $\left(V_{1}\right)$ with an amplitude of $V_{\mathrm{DD}}$, an offset of $V_{\mathrm{DD}} / 2$, and a period of $\left.T_{0}\left(=1 / f_{0}\right) ; 2\right)$ a shifting and AC-coupled circuit that generates a voltage $V_{2}$ equal to $V_{\mathrm{DD}} / 2$ plus the AM signal; 3) a voltage divider made by $R_{2}$ and $R_{3}$ yielding a low voltage $\left(V_{3}\right)$; 4$)$ a $2: 1$ analog multiplexer (MUX) that provides an output voltage $\left(V_{4}\right)$ equal to either $V_{2}$ or $\left.V_{3} ; 5\right)$ a comparator (CMP) that compares $V_{1}$ and $V_{4}$ and offers a square-wave output voltage $\left(V_{5}\right)$ whose period equals $T$; and 6) a digital timer that measures $T$ from rising to rising edge. In addition, the position of the MUX depends on the logic level of $V_{5}$ : position " 0 " when $V_{5}$ is low, and position " 1 " when $V_{5}$ is high.

The key element in Fig. 1 to satisfy the assumption indicated in the $2^{\text {nd }}$ paragraph of Section II is the MUX. This is moved to position " 1 " once the first positive crossing happens, where a positive (negative) crossing is here when $V_{1}$ becomes higher (lower) than $V_{4}$. Since $V_{3}$ is a low-value voltage (e.g. 50 or $100 \mathrm{mV}$ ), no extra voltage crossings will appear between $V_{1}$ and $V_{4}$, thus making the models developed in [10] useful for (almost) any value of $A_{\mathrm{s}}$ within the supply voltage levels. The lower $V_{3}$, the higher the maximum amplitude that can be demodulated.

The operating principle of the circuit in Fig. 1 is shown in more detail in Fig. 2. Initially, the MUX is at position " 0 " so

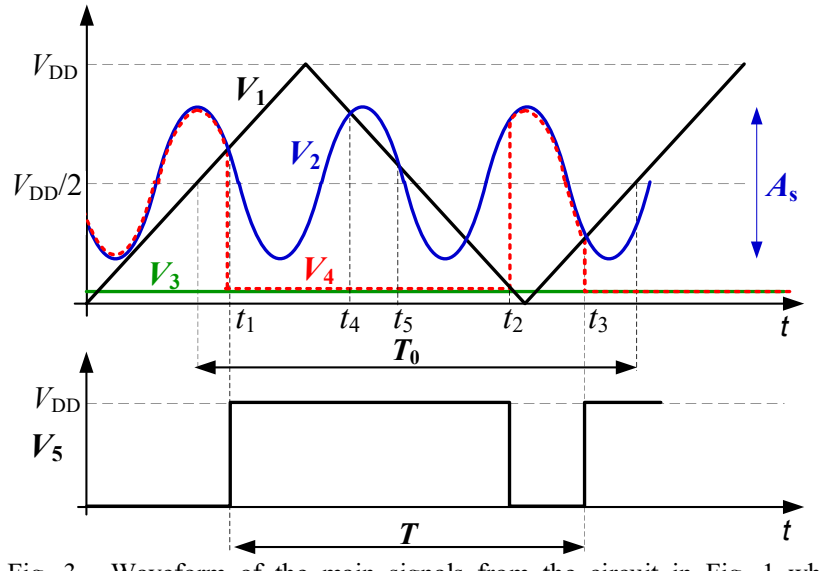

Fig. 3. Waveform of the main signals from the circuit in Fig. 1 when demodulating an AM signal with both $A_{\mathrm{s}}$ and $f_{\mathrm{c}}$ higher than those in Fig. 2.

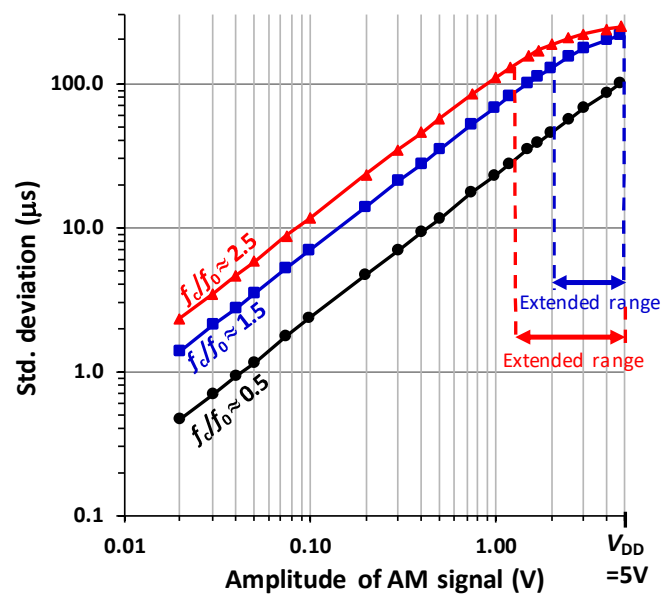

Fig. 4. Experimental values of STD versus the peak-to-peak amplitude of the AM signal for $f_{\mathrm{c}} / f_{0} \approx 0.5,1.5$, and 2.5 .

that $V_{1}$ is compared to $V_{2}$. At instant $t_{1}, V_{1}$ becomes higher than $V_{2}$ and, then, $V_{5}$ swaps to a high level that implies the start of the period measurement and the change of the position of the MUX. From now on, $V_{1}$ is compared to $V_{3}$, which is a low value. Afterwards, at instant $t_{2}, V_{1}$ becomes lower than $V_{3}$ and, hence, $V_{5}$ swaps to a low level. This moves the MUX to position " 0 ", thus comparing again $V_{1}$ and $V_{2}$. Then, at instant $t_{3}$, there is another crossing between $V_{1}$ and $V_{2}$, which causes again a low-to-high transition in $V_{5}$ that stops the measurement. In Fig. 2, the result is $T$ instead of $T_{0}$ due to the interference effects caused by the AM signal. Of course, if $A_{\mathrm{s}}=0$ then $T=T_{0}$.

The need of the MUX to avoid aberrant measurements can be better understood through the example shown in Fig. 3, where the AM signal has higher values of both $A_{\mathrm{s}}$ and $f_{\mathrm{c}}$. In such a case, if the MUX was not moved to position " 1 " after $t_{1}$, the $V_{1}-V_{2}$ comparison would have non-expected crossings (for instance, at instants $t_{4}$ and $t_{5}$ ) that would generate an aberrant value of $T$. Thanks to the MUX, any potential $V_{1}-V_{2}$ crossing from $t_{1}$ to $t_{2}$ does not affect the measurement.

\section{EXPERIMENTAL RESULTS}

The circuit in Fig. 1 has been proven using the following materials. A two-channel waveform generator (Keysight 33510B) provided the triangular and AM sinusoidal signals 


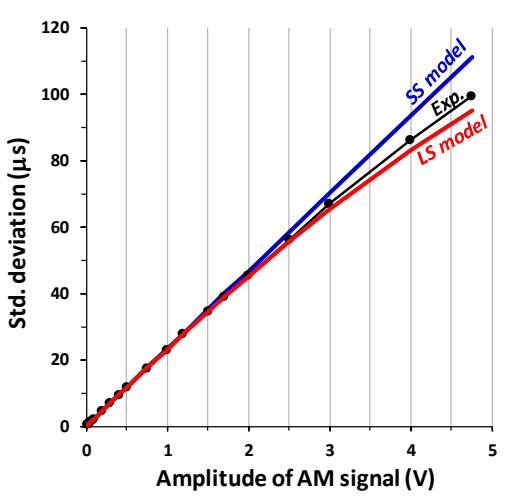

(a)

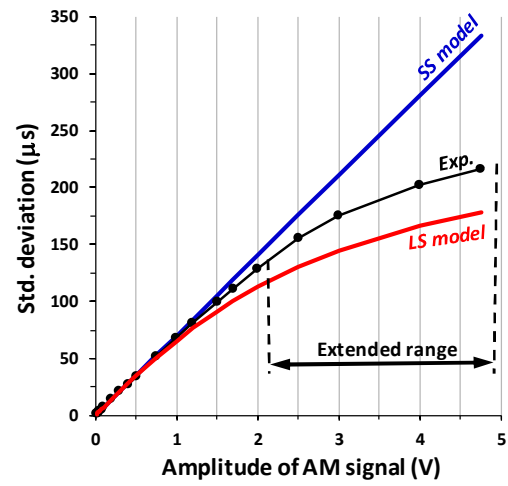

(b)

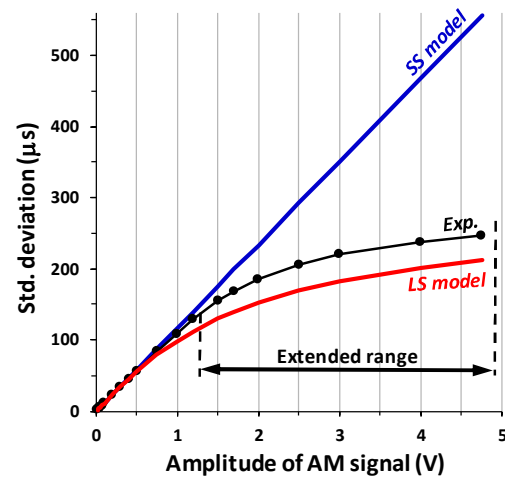

(c)

Fig. 5. Theoretical and experimental values of STD versus the peak-to-peak amplitude of the AM signal for (a) $f_{\mathrm{c}} / f_{0} \approx 0.5,(\mathrm{~b}) f_{\mathrm{c}} / f_{0} \approx 1.5$, and $(\mathrm{c}) f_{\mathrm{c}} / f_{0} \approx 2.5$. The second-degree polynomial fitted to the experimental data has the following coefficients: $a_{0}=-0.15, a_{1}=24.17$, and $a_{2}=-0.67$ in Fig. 5(a); $a_{0}=-1.45$, $a_{1}=78.51$, and $a_{2}=-6.84$ in Fig. 5(b); and $a_{0}=0.71, a_{1}=121.20$, and $a_{2}=-14.97$ in Fig. 5(c).

applying $f_{\mathrm{c}} / f_{0}=(m+0.5)(1+\Delta)$ with $\Delta=-0.67 \%$ and $m=0$, 1 , and 2 . The different ratios were obtained by changing $f_{0}$ and keeping $f_{\mathrm{c}}$ constant to $1500 \mathrm{~Hz}$, which is in the range of typical exciting frequencies for inductive sensors [12]. The selected values of $\Delta$ and $f_{\mathrm{c}}$ provided an alias component of $10 \mathrm{~Hz}$ that involves a measuring time of $100 \mathrm{~ms}$. Two commercial chips (ADG719 and AD8561 from Analog Devices) were employed as MUX and CMP, respectively. A bench-top universal counter (Agilent 53220A) measured the period of the CMP output. The resistors $R_{2}$ and $R_{3}$ were chosen to have $V_{3} \approx 100 \mathrm{mV}$ at $V_{\mathrm{DD}}=5 \mathrm{~V}$.

Fig. 4 shows how the STD of the period measurements depended on $A_{\mathrm{S}}$ for the different $f_{\mathrm{c}} / f_{0}$ under test. The higher $A_{\mathrm{s}}$, the higher STD, as expected. Moreover, histograms of 10000 period readings did not show any aberrant measurement, even for values of $A_{\mathrm{s}}$ almost equal to $V_{\mathrm{DD}}$. This clearly extended the operating range of the timer-based demodulator, as graphically indicated in Fig. 4. To be precise, the operating range was more than two and four times wider than that presented in [10] for $f_{\mathrm{c}} / f_{0} \approx 1.5$ and 2.5 , respectively. For the case $f_{\mathrm{c}} / f_{0} \approx 0.5$, the results were very similar to those shown in [10].

The experimental data in Fig. 4 is separately represented in Fig. 5 on a linear scale and together with the results predicted by the SS and LS models developed in [10]. The SS model satisfactorily predicts the response of the demodulator up to $A_{\mathrm{s}} \approx 2,1.5$, and $1 \mathrm{~V}$ for $f_{\mathrm{c}} / f_{0} \approx 0.5,1.5$, and 2.5 , respectively. Beyond this value, the input-output characteristic suffers from nonlinearity effects that are well predicted by the LS model. At the worst point (i.e. at $A_{\mathrm{s}} \approx V_{\mathrm{DD}}$ ), the difference between the experimental value of STD and that predicted by LS is $4 \%$, $17 \%$, and $14 \%$ in Figs. 5(a), (b), and (c), respectively. A second-degree polynomial (i.e. STD $=a_{0}+a_{1} A_{\mathrm{s}}+a_{2} A_{\mathrm{s}}^{2}$ ) can be accurately fitted to the experimental data shown in Fig. 5 with a coefficient of determination $\left(R^{2}\right)$ higher than 0.999 , thus facilitating the circuit calibration.

\section{CONCLUSIONS}

This paper has proposed a novel timer-based demodulator for AM sensor signals. In comparison with the state of the art, the novel circuit incorporates a simple but practical MUX that avoids aberrant measurements. As a consequence of that, the operating range of the circuit has been extended all the way between the supply voltage levels, which means up to four times wider than that of the previous topology. Experimental results have shown that the input-output characteristic in such a wider operating range is not linear, but it can be accurately modelled by a second-degree polynomial.

\section{REFERENCES}

[1] F. Reverter and Ò. Casas, "Interfacing differential resistive sensors to microcontrollers: a direct approach," IEEE Trans. Instrum. Meas., vol. 58 , no. 10 , pp. 3405-3410, Oct. 2009.

[2] O. Oballe-Peinado, F. Vidal-Verdú, J. A. Sánchez-Durán, J. CastellanosRamos, and J. A. Hidalgo-López, "Accuracy and resolution analysis of a direct resistive sensor array to FPGA interface," Sensors, vol. 16, no. 2, pp. 181-196, 2016.

[3] P. R. Nagarajan, B. George, and V. J. Kumar, "Improved single-element resistive sensor-to-microcontroller interface," IEEE Trans. Instrum. Meas., vol. 66, no. 10, pp. 2736-2744, Oct. 2017.

[4] F. Reverter and Oे. Casas, "Interfacing differential capacitive sensors to microcontrollers: a direct approach," IEEE Trans. Instrum. Meas., vol. 59, no. 10, pp. 2763-2769, Oct. 2010.

[5] J. Pelegrí-Sebastiá, E. García-Breijo, J. Ibáñez, T. Sogorb, N. LaguardaMiro, and J. Garrigues, "Low-cost capacitive humidity sensor for application within flexible RFID labels based on microcontroller systems," IEEE Trans. Instrum. Meas., vol. 61, no. 2, pp. 545-553, Feb. 2012.

[6] Z. Kokolanski, J. Jordana, M. Gasulla, V. Dimcev, and F. Reverter, "Direct inductive sensor-to-microcontroller interface circuit," Sens. Actuators A, vol. 224, pp. 185-191, 2015.

[7] N. Ramadoss and B. George, "A simple microcontroller based digitizer for differential inductive sensors," in Proc. IEEE Int. Instrum. Meas. Technol. Conf., pp. 148-153, 2015.

[8] L. Bengtsson, "Direct analog-to-microcontroller interfacing," Sens. Actuators A, vol. 179, pp. 105-113, 2012.

[9] L. Dutta, A. Hazarika, and M. Bhuyan, "Nonlinearity compensation of DIC-based multi-sensor measurement," Measurement, vol. 126, pp. 1321, Oct. 2018.

[10] F. Reverter and M. Gasulla, "Timer-based demodulator for AM sensor signals applied to an inductive displacement sensor," IEEE Trans. Instrum. Meas., vol. 66, no. 10, pp. 2780- 2788, Oct. 2017.

[11] F. Reverter, M. Gasulla, and R. Pallàs-Areny, "Analysis of interference effects on period-to-digital conversions," Meas. Sci. Technol., vol. 16, pp. 2261-2264, 2005.

[12] N. Mandal, B. Kumar, R. Sarkar, and S. C. Bera, "Design of a flow transmitter using an improved inductance bridge network and rotameter as sensor," IEEE Trans. Instrum. Meas., vol. 63, no. 12, pp. 3127-3136, Dec. 2014. 